\title{
ON THE PLANE PROBLEM OF A PERFECT PLASTIC BODY ${ }^{1}$
}

\author{
BY \\ HILDA GEIRINGER \\ Wheaton College
}

In the equilibrium problem of the isotropic ideal plane plastic body it is assumed that the state of stress is restricted to a one-dimensional variety $F\left(\sigma_{1}, \sigma_{2}\right)=0$ where $\sigma_{i}(i=1,2)$ denote the principal stresses; moreover, the two equilibrium conditions hold. This problem may be derived in several ways from the complete three-dimensional problem of the perfect plastic body; in particular the assumption $\sigma_{3}=\sigma_{z}=0, \partial \cdots / \partial z=$ 0 leads to it without the need for consideration of any three-dimensional relation between strain-rates and stresses. However, as long as $F\left(\sigma_{1}, \sigma_{2}\right)$ is not specified the relation of the plane problem to the three-dimensional problem need not be considered.

Important particular assumptions for the yield condition, $F\left(\sigma_{1}, \sigma_{2}\right)=0$, are the v. Mises condition of constant shear energy and the Saint Venant condition of constant maximum shearing stress. For the first, $F$ is given by

$$
\sigma_{1}^{2}-\sigma_{1} \sigma_{2}+\sigma_{2}^{2}=4 K^{2},
$$

the second states that $|\tau|_{\max }=\left|\sigma_{1}-\sigma_{2}\right| / 2=K$, if $\sigma_{1}$ and $\sigma_{2}$ have opposite signs, while otherwise either $\sigma_{1}=2 K$, or $\sigma_{2}=2 K$. Hence

$$
\begin{array}{rlrlrl}
\left|\sigma_{1}-\sigma_{2}\right| & =2 K, & & \text { if } & \sigma_{1} \sigma_{2} & \leqq 0, \\
& =4 K-\left|\sigma_{1}+\sigma_{2}\right|, & \text { if } & \sigma_{1} \sigma_{2} & \geqq 0 .
\end{array}
$$

These forms of $F$ are derived from corresponding three-dimensional conditions by means of the afore-mentioned assumption of "plane stress", $\sigma_{3}=0, \partial \cdots / \partial z=0$.

The problem most widely investigated is that with the yield condition $\left(\sigma_{1}-\sigma_{2}\right)^{2}=$ $4 K^{2}$ as in the first Eq. (2). It is distinguished by its comparative mathematical simplicity; moreover, this same yield condition appears in "plane strain", as both v. Mises' and Saint Venant's condition.

The general plane problem defined by the two equilibrium equations and some yield condition has been considered by Sokolovsky [6a, b] for the two conditions (1) and (2); and for general yield conditions by v. Mises [4a], Geiringer [1], and the Brown University group, (see P. G. Hodge [2], Chap. VIII). As in the case of "plane strain" one can-in several ways - derive from the basic equations a linear system by a change of variables, somehow analogous to the Chaplygin transformation in the theory of compressible fluids; the plane of the new independent variables called "stress graph" by v. Mises, corresponds then to the hodograph plane, the yield condition to the "adiabatic" condition. It is intended to follow up this analogy in several directions.

First we establish linear differential equations of second order for certain magnitudes which may serve as coordinates in the physical plane; such an equation is amenable to well-known analytic methods. The coefficients depend, of course, on the yield condition;

${ }^{1}$ Received Oct. 13, 1950. The main results of this paper have been communicated in a talk given at the Second Symposium on Plasticity, Providence, April 1949, and later briefly summarized in three abstracts of papers presented by title at the October 1949 meeting of the A.M.S. [1]. 
we consider particularly conditions (1) and (2) and the new "parabola condition" recently proposed [4a] by v. Mises. Next the characteristics are investigated, in general, and for the afore-mentioned yield conditions; here most of our results while definitely more simple are essentially identical with corresponding results of Sokolovsky [6a], v. Mises [4a], Hodge [2]. Finally, simple wave solutions are considered. The stress distributions are determined and discussed; cross characteristics and lines of principal stress are computed. In each of the indicated directions one can go much further, this paper thus being of an introductory character; solutions of concrete problems have not been included.

1. The stress graph. Following v. Mises we express the plasticity condition in terms of an appropriate parameter, $s$ :

$$
F\left(\sigma_{1}, \sigma_{2}\right)=0, \quad \sigma_{1}=\sigma_{1}(s), \quad \sigma_{2}=\sigma_{2}(s) .
$$

Let us assume from now on that $\sigma_{2} \geqq \sigma_{1}$. It is often convenient to take for $s$ the average stress $\left(\sigma_{1}+\sigma_{2}\right) / 2 K$. Denoting by $\theta$ the angle between the positive $x$ direction and the $\xi$ direction, i.e., the (positive) first principal direction, we take $s$ and $\theta$ as new variables which determine the stress tensor at each point. The plane in which $s$ and $\theta$ are polar coordinates is called stress graph by v. Mises. Denote by $\partial \cdots / \partial \xi$ and $\partial \cdots / \partial \eta$ the directional derivatives in the first and second principal direction, respectively, then equilibrium conditions are equivalent to the system (3):

$$
\frac{\partial \sigma_{1}}{\partial \xi}=\left(\sigma_{2}-\sigma_{1}\right) \frac{\partial \theta}{\partial \eta}, \quad \frac{\partial \sigma_{2}}{\partial \eta}=\left(\sigma_{2}-\sigma_{1}\right) \frac{\partial \theta}{\partial \xi} .
$$

With the use of (3) and the abbreviations, $d \sigma_{1} / d s=\sigma_{1}^{\prime}(s)$, etc.,

$$
\left(\sigma_{2}-\sigma_{1}\right) / \sigma_{1}^{\prime}=f(s), \quad\left(\sigma_{2}-\sigma_{1}\right) / \sigma_{2}^{\prime}=g(s),
$$

(4) yields the reducible equations

$$
\frac{\partial s}{\partial \xi}=f(s) \frac{\partial \theta}{\partial \eta}, \quad \frac{\partial s}{\partial \eta}=g(s) \frac{\partial \theta}{\partial \xi} .
$$

At every point where the Jacobian $j=\partial(s, \theta) / \partial(\xi, \eta)$ is different from zero in (6) the dependent and independent variables may be interchanged, to yield v. Mises' linear equations

$$
\frac{\partial \eta}{\partial \theta}=f(s) \frac{\partial \xi}{\partial s}, \quad \frac{\partial \xi}{\partial \theta}=g(s) \frac{\partial \eta}{\partial s} .
$$

2. Linear differential equations of second order. In Eq. (6) $\xi$ and $\eta$ are not coordinates; it is, however, possible to derive from (7) in various ways systems of equations for quantities which may serve as coordinates in the physical plane. If, e.g.,

$$
X=x \cos \theta+y \sin \theta, \quad Y=y \cos \theta-x \sin \theta,
$$

we find the system of linear equations of first order:

$$
\frac{\partial X}{\partial \theta}=g(s) \frac{\partial Y}{\partial s}+Y, \quad \frac{\partial Y}{\partial \theta}=f(s) \frac{\partial X}{\partial s}-X .
$$


From (9) we obtain the equations of second order:

$$
\begin{aligned}
& \frac{\partial^{2} X}{\partial \theta^{2}}-f g \frac{\partial^{2} X}{\partial s^{2}}=-X+\frac{\partial X}{\partial s}\left(f^{\prime} g+f-g\right), \\
& \frac{\partial^{2} Y}{\partial \theta^{2}}-f g \frac{\partial^{2} Y}{\partial s^{2}}=-Y+\frac{\partial Y}{\partial s}\left(g^{\prime} f+f-g\right) .
\end{aligned}
$$

These equations ${ }^{1}$ are hyperbolic, or elliptic, according to whether $f g \gtrless 0$ (see (5)) or:

$$
\frac{d \sigma_{1}}{d s} \frac{d \sigma_{2}}{d s} \gtrless 0 ;
$$

they are parabolic if either $f g$ or its reciprocal value equals zero. If a solution $X(s, \theta)$ of $\left(10^{1}\right)$ has been found, the corresponding $Y(s, \theta)$ follows from $\left(9^{2}\right)$ and the coordinates $x, y$ follow from (8) in terms of $s$ and $\theta$ without further integration; thus $s$ and $\theta$ are defined in terms of $x$ and $y$.

Next, we satisfy the first Eq. (9) by a function $\psi(s, \theta)$ such that

$$
\frac{\partial \psi}{\partial s}=\frac{X}{g(s) k(s)}, \quad \frac{\partial \psi}{\partial \theta}=\frac{Y}{k(s)},
$$

where $k$ satisfies the condition $g(s) k^{\prime}(s)+k(s)=0$.

Substituting $\psi$ into the second Eq. (9), we find

$$
\frac{\partial^{2} \psi}{\partial \theta^{2}}-f g \frac{\partial^{2} \psi}{\partial s^{2}}=\frac{\partial \psi}{\partial s}\left(f g^{\prime}-f-g\right) .
$$

Also with

$$
\frac{\partial \varphi}{\partial s}=\frac{Y}{f(s) h(s)}, \quad \frac{\partial \varphi}{\partial \theta}=\frac{X}{h(s)}, \quad f(s) h^{\prime}(s)-h(s)=0
$$

the equation

$$
\frac{\partial^{2} \varphi}{\partial \theta^{2}}-f g \frac{\partial^{2} \varphi}{\partial s^{2}}=\frac{\partial \varphi}{\partial s}\left(g f^{\prime}+g+f\right)
$$

follows in the same manner.

For finding integrals of (10) or (13), or (15) two principal methods are available: (a) Bergman's operator method may be adapted to our problem; (b) the classical way of expansion in series seems promising. In these ways regular solutions as well as solutions with appropriate singularities may be obtained. Because of the linearity of (10) solutions may be superimposed. On the other hand, the difficulties encountered in actual boundary value problems, where the boundary conditions are given in the physical plane, are well known.

3. Particular yield conditions. We consider v. Mises' "quadratic" condition (1), the the "hexagonal" condition of Saint Venant, and v. Mises' new "parabola condition".

${ }^{1}$ Equations (10) are simpler than Eq. (19) of v. Mises [4a] because $X$ and $Y$ rather than $x$ and $y$ have been chosen as dependent variables. 
In the $\sigma_{1}-\sigma_{2}$-plane (Fig. 1) the Mises limit (1) is represented by the ellipse

$$
\begin{gathered}
s=\frac{1}{2 K}\left(\sigma_{1}+\sigma_{2}\right), \quad(-2 \leqq s \leqq+2), \\
\sigma_{1}=K\left[s \pm\left(\frac{4-s^{2}}{3}\right)^{1 / 2}\right], \quad \sigma_{2}=K\left[s \mp\left(\frac{4-s^{2}}{3}\right)^{1 / 2}\right],
\end{gathered}
$$

where the upper signs apply to the right, the lower to the left part of the ellipse. Then

$$
\sigma_{1}^{\prime} \sigma_{2}^{\prime}=\frac{4 K^{2}}{3} \frac{3-s^{2}}{4-s^{2}}, \quad f g=\frac{\left(\sigma_{2}-\sigma_{1}\right)^{2}}{\sigma_{2}^{\prime} \sigma_{1}^{\prime}}=\frac{\left(4-s^{2}\right)^{2}}{3-s^{2}} .
$$

It is seen that $f g>0$ for $s^{2}<3, f g<0$ for $s^{2}>3$ while $f g \rightarrow \pm \infty$ as $s^{2} \rightarrow 3$. Since we assume $\sigma_{2} \geqq \sigma_{1}$ we are concerned merely with the left part of the ellipse; the hyperbolic case corresponds to the interval $-\sqrt{3}<s<+\sqrt{3}$; to $s=+\sqrt{3}$ and $s=-\sqrt{3}$
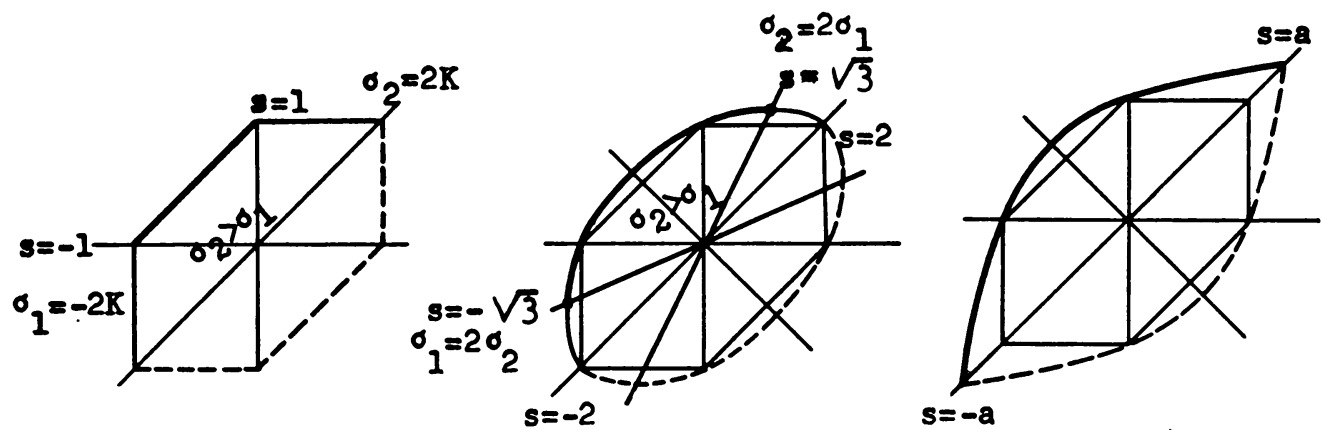

FIG. 1. Yield conditions.

correspond the "sonic points" $\sigma_{2}=2 \sigma_{1}=4 K / \sqrt{3}$ and $\sigma_{1}=2 \sigma_{2}=-4 K / \sqrt{3}$. In the physical plane the "sonic line" separates the domain of hyperbolic solutions from the elliptic domain; its image in the stress graph is the circle about the origin with radius $s=\sqrt{3}$. The representation of the "hyperbolic part", for $\sigma_{2}>\sigma_{1}$ is:

$$
\sigma_{1}=K\left[s-\left(\frac{4-s^{2}}{3}\right)^{1 / 2}\right], \quad \sigma_{2}=K\left[s+\left(\frac{4-s^{2}}{3}\right)^{1 / 2}\right], \quad|s|<\sqrt{3} .
$$

The complete Saint-Venant limit (2) is represented by a hexagon inscribed in the ellipse (16). With $s=\left(\sigma_{1}+\sigma_{2}\right) / 2 K$, the representation of the "hyperbolic part" of this limit is

and

$$
\sigma_{1}=K(s-1), \quad \sigma_{2}=K(s+1), \quad|s|<1
$$

$$
\sigma_{1}^{\prime} \sigma_{2}^{\prime}=K^{2}, \quad f g=4 .
$$

The vertical and horizontal lines of the hexagon correspond to parabolic domains, where either $\sigma_{1}$ or $\sigma_{2}$ are constant.

The "parabola limit" consists of two branches of parabolas passing through four corners of the Saint Venant hexagon:

$$
\frac{\sigma_{2}-\sigma_{1}}{K}= \pm \frac{1}{a}\left[a^{2}-\left(\frac{\sigma_{1}+\sigma_{2}}{2 K}\right)^{2}\right]
$$


where $a=1+\sqrt{2}$, the plus sign applying to the left, the minus to the right branch. The parametric representation for $\sigma_{2}>\sigma_{1}$ is (with our parameter)

$$
\sigma_{1}=K s-\frac{K}{2 a}\left(a^{2}-s^{2}\right), \quad \sigma_{2}=K s+\frac{K}{2 a}\left(a^{2}-s^{2}\right), \quad|s|<a
$$

and

$$
f=a-s, \quad g=a+s, \quad f g=a^{2}-s^{2} .
$$

It is seen that, with the only exception of the points $s= \pm a$, where $f g=0$, the problem is everywhere hyperbolic.

As an application let us consider the second order equations of the preceding section for the parabola limit, transforming them at the same time to standard forms. With

$$
\frac{d s}{d u}=\left(a^{2}-s^{2}\right)^{1 / 2}, \quad s=a \sin u, \quad u=\arcsin s / a
$$

and putting $X(s, \theta)=\bar{X}(u, \theta)$, and $A(u)=(1+2 \sin u) / \cos u$, Eq. (10) becomes

$$
\frac{\partial^{2} \bar{X}}{\partial \theta^{2}}-\frac{\partial^{2} \bar{X}}{\partial u^{2}}+\bar{X} \cdot A(u)=0, \quad|u|<\frac{\pi}{2} \text {. }
$$

Next with $p(u)=[\cos u(1-\sin u)]^{-1 / 2}$, where $A p-2 p^{\prime}=0$, it assumes the form

$$
\frac{\partial^{2} Z}{\partial \theta^{2}}-\frac{\partial^{2} Z}{\partial u^{2}}+Z \frac{1+2 \sin u}{4 \cos ^{2} u}=0 .
$$

This is an equation in standard form which may be treated in various manners.

Also, with $\psi(s, \theta)=\bar{\psi}(u, \theta)$, Eq. (13) takes the form

$$
\frac{\partial^{2} \bar{\psi}}{\partial \theta^{2}}-\frac{\partial^{2} \bar{\psi}}{\partial u^{2}}+\frac{\partial \bar{\psi}}{\partial u} \cdot \frac{1}{\cos u}=0,
$$

and putting $\bar{\psi}(u, \theta)=r(u) R(u, \theta)$ where

$$
r(u)=\left(\frac{1+\sin u}{\cos u}\right)^{1 / 2}, \quad 2 r^{\prime} \cos u-r=0,
$$

we obtain

$$
\frac{\partial^{2} R}{\partial \theta^{2}}-\frac{\partial^{2} R}{\partial u^{2}}+R \frac{1-2 \sin u}{4 \cos ^{2} u}=0 .
$$

Note the difference between (10a) and (13b).

As a second application, we give an example of particular solutions of Eqs. (13). Set

$$
\psi(s, \theta)=P(s) Q(\theta) .
$$

One obtains in the usual way, denoting by $\lambda$ a constant, and using (22):

$$
\left(a^{2}-s^{2}\right) P^{\prime \prime}-(a+s) P^{\prime}-\lambda P=0 .
$$

Introducing the new variables $u$ and $U$ by

$$
s=a(2 u-1), \quad \frac{d P}{d s}=\frac{1}{2 a} \frac{d U}{d u}, \quad \frac{d^{2} P}{d s^{2}}=\frac{1}{4 a^{2}} \frac{d^{2} U}{d u^{2}},
$$


we find

$$
u(1-u) \frac{d^{2} U}{d u^{2}}-u \frac{d U}{d u}-U=0,
$$

which is a hypergeometric equation. Solutions of (24) are well known; with $Q=C \sin$ $n\left(\theta-\theta_{0}\right)$, we may thus find solutions of the form (23), which may be superimposed.

4. Characteristics. The characteristics of the reducible equations (6) are easily found. If $\varphi$ denotes the angle of a characteristic direction with the $\xi$-direction, we find by standard methods:

$$
\tan ^{2} \varphi=\frac{f}{g}, \quad \tan \varphi=\frac{d \eta}{d \xi}= \pm\left(\frac{f}{g}\right)^{1 / 2} .
$$

In the hyperbolic domain, $f$ and $g$ have the same sign; thus, at each point of this domain, there are two distinct directions making equal angles with the $\xi$-direction. We
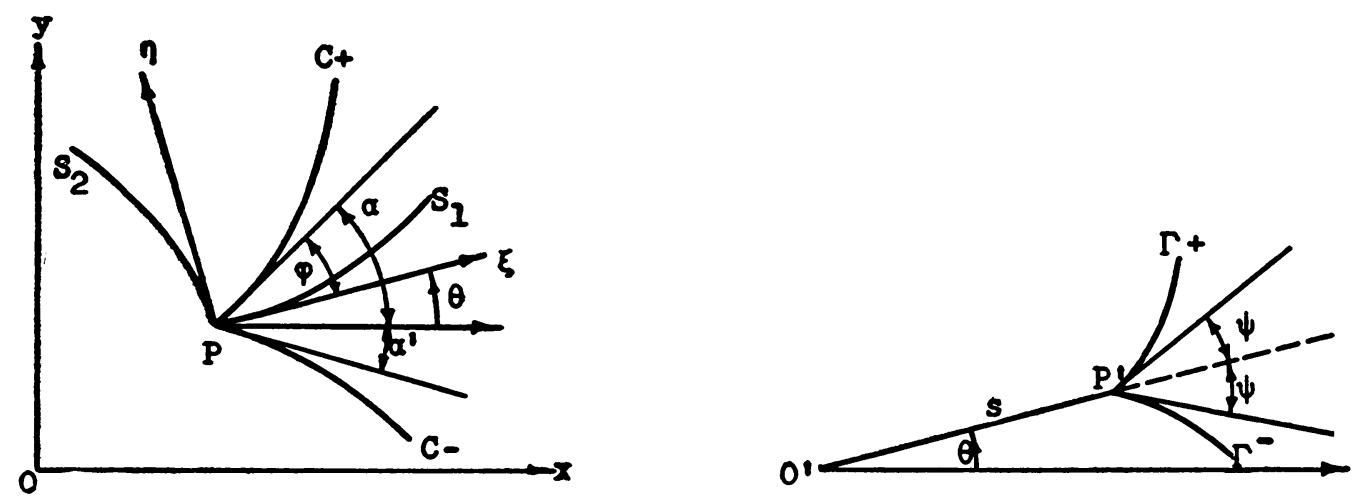

FIG. 2. Characteristics in physical plane and in stress plane.

denote by $C^{+},\left(C^{-}\right)$the characteristics making the angles $+\varphi,(-\varphi)$ with the $\xi$-direction, and by

$$
\alpha=\theta+\varphi, \quad \alpha^{\prime}=\theta-\varphi
$$

the angles of $C^{+}$and $C^{-}$with the $x$-axis (Fig. 2). Then, with $d y / d x$ for the direction coefficient of a characteristic, we have

$$
\frac{d y}{d x}=\tan (\theta \pm \varphi)=\frac{\tan \theta \pm(f / g)^{1 / 2}}{1 \mp \tan \theta \cdot(f / g)^{1 / 2}}
$$

the upper (lower) sign applying to $C^{+}$(to $C^{-}$). If a solution $s=s(x y), \theta=\theta(x, y)$ is introduced into $\left(26^{\prime}\right)$, this becomes an ordinary differential equation for the determination of the characteristic curves.

Note also that, according to (3), (5), and (25) with $F_{i}=\partial F / \partial \sigma_{i}$,

$$
\tan ^{2} \varphi=\frac{\sigma_{2}^{\prime}}{\sigma_{1}^{\prime}}=\frac{d \sigma_{2}}{d \sigma_{1}}=-\frac{F_{1}}{F_{2}}
$$

depending merely on the yield condition: $F\left(\sigma_{1}, \sigma_{2}\right)=0$. For the "quadratic" condition, 
for example, this gives $\tan ^{2} \varphi=\left(\sigma_{2}-2 \sigma_{1}\right) /\left(2 \sigma_{2}-\sigma_{1}\right)$, showing that along the "sonic line" $\varphi$ equals 0 or $\pi / 2$.

From the general definition of characteristics it follows that a relation exists between the derivatives of the dependent variables along a characteristic direction. To find this, multiply the first term in the first Eq. (6) by $\cos \varphi$, the second by $(g / f)^{1 / 2} \sin \varphi$; in the second Eq. (6) multiply the first term by $\sin \varphi$, the second by $(f / g)^{1 / 2} \cos \varphi$ and add. ${ }^{1}$ Thus,

$$
\frac{\partial s}{\partial \xi} \cos \varphi+\frac{\partial s}{\partial \eta} \sin \varphi=(f g)^{1 / 2} \cdot\left(\frac{\partial \theta}{\partial \xi} \cos \varphi+\frac{\partial \theta}{\partial \eta} \sin \varphi\right) .
$$

Denoting by $\partial \cdots / \partial l$ differentiation in a characteristic direction, we obtain the desired relations:

$$
\frac{\partial s}{\partial l^{+}}=(f g)^{1 / 2} \frac{\partial \theta}{\partial l^{+}}, \quad \frac{\partial s}{\partial l^{-}}=-(f g)^{1 / 2} \frac{\partial \theta}{\partial l^{-}} .
$$

These relations between the derivatives of $s$ and $\theta$ along $C^{+}$or $C^{-}$do not depend on the validity of the transformation (7).

It follows from equation (10) or (15) that for the fixed characteristics $\Gamma$ in the stress graph:

$$
\left(\frac{d \theta}{d s}\right)^{2}=(f g)^{-1}, \quad \frac{d \theta}{d s}= \pm(f g)^{-1 / 2}
$$

Writing:

$$
\int^{s}(f g)^{-1 / 2} d s=F(s)
$$

(with an adequate lower limit), we obtain the equations of the two families of fixed characteristics, $\Gamma^{+}$and $\Gamma^{-}$:

$$
\theta-F(s)=\text { const, } \quad \theta+F(s)=\text { const. }
$$

Equation (28) may also be derived from (7) in the same way as (24) was derived from (6). Then (27) shows that (as always) the characteristics in the two planes which are mapped onto each other (here the $x, y$ plane and the $s, \theta$ plane) correspond to each other, here $C^{+}$to $\Gamma^{+}, C^{-}$to $\Gamma^{-}$. We see from (28) that $\Gamma^{+}$and $\Gamma^{-}$make equal angles $+\psi$ and $-\psi$ with the radius vector and $\tan \psi=s d \theta / d s$ (Fig. 2).

We consider here in general the hyperbolic case; let us, however, just say a word about the parabolic case where $f / g=\sigma_{2}^{\prime} / \sigma_{1}^{\prime}$ is either zero or infinite. If $\sigma_{2}^{\prime}=0\left(\sigma_{1}^{\prime}=0\right)$ the angle $\varphi=0,(\varphi=\pi / 2)$ and the one existing characteristic direction coincides with the direction of first (second) principal stress. In either case $\psi=0, d \theta / d s=0$. It follows from (28) that in a parabolic region the characteristics $\Gamma$ in the stress graph are radii through $O^{\prime}$, and from $\left(26^{\prime}\right)$ by integration that the characteristics in the physical plane form likewise a set of straight lines: $y=x \tan \theta+G(\theta)$ if $\sigma_{2}^{\prime}=0$.

5. Characteristics. Continuation. The $\Gamma$-curves in the stress graph have been investigated by $\mathrm{v}$. Mises for the three yield conditions and sketched with $s$ and $\theta$ as polar coordinates. The formulas are:

\footnotetext{
IIn the hyperbolic case $g / f$ is nowhere zero or infinite.
} 
Quadratic condition (v. Mises)

$$
\begin{aligned}
\frac{d \theta}{d s} & = \pm \frac{\left(3-s^{2}\right)^{1 / 2}}{4-s^{2}} \\
\pm \theta & =\arctan \left[s\left(3-s^{2}\right)^{-1 / 2}\right]-\frac{1}{2} \arctan \left[\frac{s}{2}\left(3-s^{2}\right)^{-1 / 2}\right]+\text { const }
\end{aligned}
$$

and for the $C$ - characteristics:

$$
\tan \varphi= \pm \frac{\left(12-3 s^{2}\right)^{1 / 2}-s}{2\left(3-s^{2}\right)^{1 / 2}} .
$$

At the "sonic points" $\tan \varphi$ is zero or infinite and $\psi=0$. The characteristics (29) are congruent curves (like the epicycloids in our analogy). In rectangular coordinates, $s$ and $\theta$, a $\Gamma^{+}$line is monotonously increasing e.g. from $-\pi / 4$ to $\pi / 4$, showing central symmetry, inflection points at $s=0, s= \pm \sqrt{2}$, and $d \theta / d s=0$ at $s= \pm \sqrt{3}$ (Fig. 3).

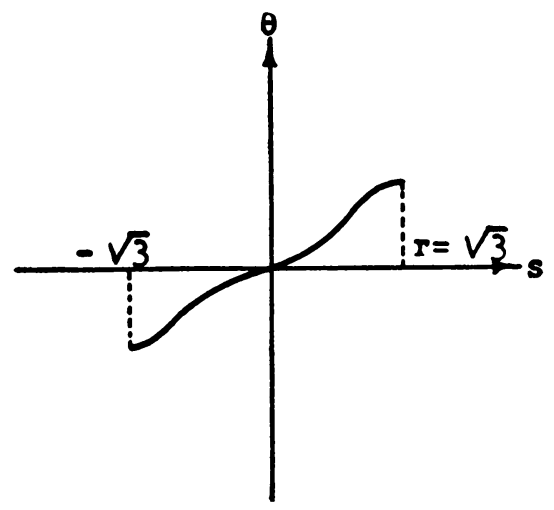

Fig. 3. $\theta=F(s)$.

Saint Venant condition. Here $d \theta / d s= \pm 1 / 2$. The $\Gamma$-characteristics in the hyperbolic area in the stress graph are ordinary spirals $\pm \theta=s / 2+$ const, or straight lines in the rectangular coordinates $s, \theta$ (in the parabolic area the unique set consists of radii through $\left.O^{\prime}\right)$; also $\tan \varphi= \pm 1, \alpha=\theta+\pi / 4, \alpha^{\prime}=\theta-\pi / 4$; it is thus seen that the characteristics in the $x y$-plane coincide with the shear lines, a result well known in the classical theory.

Parabola condition.

$$
\frac{d \theta}{d s}= \pm\left(a^{2}-s^{2}\right)^{-1 / 2}, \quad \theta= \pm \arcsin \frac{s}{a}+\text { const. }
$$

These are sine-lines $s= \pm a \sin \left(\theta-\theta_{0}\right)$ if interpreted in rectangular coordinates and circles through the origin in polar coordinates. Here,

$$
\tan \varphi= \pm\left(\frac{a-s}{a+s}\right)^{1 / 2}
$$

We finish this section by a remark on the approximate solution of the classical initial value problems in the hyperbolic domain. In the Cauchy problem, on a smooth are of curve, $K$, values of $s$ and $\theta$ are prescribed (with continuous second derivatives) so that 
$K$ has nowhere a characteristic direction, i.e. that $\left(26^{\prime}\right)$ does not hold. These data assure uniqueness of solution in the characteristic quadrangle determined by both, $\mathrm{C}^{+}$and $\mathrm{C}^{-}$, at both endpoints of $K$. There exists certainly a solution in the neighborhood of $K$ which on $K$ takes on the prescribed values. It can be obtained approximately by using the fixed net of $\Gamma$-characteristics (adjacent to the image $K^{\prime}$ of $K$ ) in order to find the $(s, \theta)$ values for corresponding lattice points in the physical plane. In general, we may then be able to continue in this way until the whole characteristic quadrangle has been filled. The procedure may be refined in various ways.

For the characteristic initial value problem of reducible equations, like (6), the data which can be arbitrarily prescribed are not the same as in the better known linear case. (This difference is often not appreciated, see [2], p. 197.) In the reducible case any arbitrarily chosen curve $C: y=y(x)$ may be a characteristic, but along this $C$ neither $s$ nor $\theta$ may be arbitrarily prescribed, since along $C$ one relation $\left(26^{\prime}\right)$ and one (28) (or rather (27)) must hold. More generally, for a curve $y=y(x)$ to be a characteristic, the three functions $y(x), s(x), \theta(x)$ must satisfy two relations: one $\left(26^{\prime}\right)$, and one (28).

For two intersecting characteristics, $C^{+}, C^{-}$the values of $s$ and $\theta$ at the point of intersection must satisfy both relations $\left(26^{\prime}\right)$; the relations (28), which merely connect $d \theta$ and $d s$ leave the values at one point unrestricted (or, in other words, the constants in

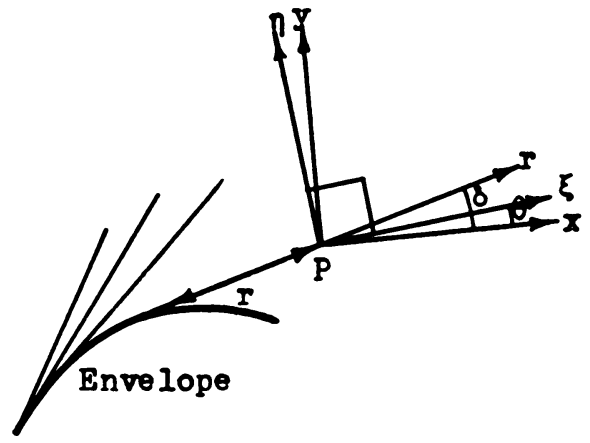

FIG. 4. Family of straight characteristics.

$\left(28^{\prime \prime}\right)$ may be determined so that the four equations $\left(26^{\prime}\right)$ and $\left(28^{\prime \prime}\right)$ are satisfied at the point of intersection). If in this way two intersecting characteristics are given, the solution in the corresponding quadrangle is uniquely determined, and it may be found step by step in a way analogous to that indicated above.

6. Simple waves. A simple wave region is a domain $D$ in the physical plane mapped onto one single characteristics, say $\Gamma_{0}^{+}$, of the $s, \theta$ plane such that to each point on $\Gamma_{0}^{+}$corresponds a straight line in $D$ carrying constant values of $s$ and $\theta$ (and consequently of $\left.\sigma_{1}, \sigma_{2}, \theta\right)$. In such a "forward wave" the straight lines are the $C^{-}$characteristics, and $\theta-F(s)$ is constant for all $(x, y)$ in $D$; all characteristics of the other family, the "cross characteristics", $C^{+}$, are mapped onto $\Gamma_{0}^{+}$. The Jacobian, $j$, of this mapping vanishes, of course. Similarly we define a "backward wave". Naturally, not all the above noted properties are needed for the definition of a simple wave. The notion includes as particular case that of constant stress tensor in $D$.

Here we start with the problem of finding solutions of Eqs. (6) such that the state of stress is constant along each straight line of an arbitrary set of lines. Denote by $\delta$ the angle 
of such a straight line, $L$, with the $x$ direction (Fig. 4). Since $\sigma_{1}, \sigma_{2}$, and $\theta$ remain constant along $L,(4)$ yields

$$
\begin{aligned}
& \sin (\theta-\delta) \frac{d \sigma_{1}}{d \delta}=\left(\sigma_{2}-\sigma_{1}\right) \cos (\theta-\delta) \frac{d \theta}{d \delta} \\
& \cos (\theta-\delta) \frac{d \sigma_{2}}{d \delta}=\left(\sigma_{2}-\sigma_{1}\right) \sin (\theta-\delta) \frac{d \theta}{d \delta}
\end{aligned}
$$

By division, we obtain

$$
\tan ^{2}(\theta-\delta)=\frac{d \sigma_{2}}{d \sigma_{1}}=\frac{-F_{1}(s)}{F_{2}(s)}
$$

as in (25). Next we multiply the first Eq. (33) by $\cos (\theta-\delta)$, the second by $\sin (\theta-\delta)$. and add:

$$
\frac{d\left(\sigma_{1}+\sigma_{2}\right)}{d \theta}=\frac{\sigma_{2}-\sigma_{1}}{\sin (\theta-\delta) \cos (\theta-\delta)} .
$$

From (35) and (34) we then find easily our Eq. (28). In fact,

$$
\begin{gathered}
d \theta=\frac{d\left(\sigma_{1}+\sigma_{2}\right)}{\sigma_{2}-\sigma_{1}} \sin (\theta-\delta) \cos (\theta-\delta)=\frac{\left(d \sigma_{2} d \sigma_{1}\right)^{1 / 2}}{\sigma_{2}-\sigma_{1}} \\
\frac{d \theta}{d s}=\frac{\left(\sigma_{1}^{\prime} \sigma_{2}^{\prime}\right)^{1 / 2}}{\sigma_{2}-\sigma_{1}}=(f g)^{-1 / 2} .
\end{gathered}
$$

Thus, if a condition (3) and a $\Gamma_{0}^{+}$are chosen we find to each $\delta$ the $s$ and $\theta$ by (28) and (34). If, however, we want to find cross characteristics, $C^{+}$, and lines of principal stress we have to consider not only $\delta$ but the whole set of straight characteristics. Let it be given in the form

$$
y=\beta x+h(\beta),
$$

where $\beta=\tan \delta=\tan (\theta-\varphi)$. The differential equation of the cross characteristics is $d y / d x=\tan (\theta+\varphi)$ and since $\theta$ and $\varphi$ both depend in a given way on $s$, and $s$ on $\beta$ we have

$$
\frac{d y}{d x}=\tan (\theta+\varphi)=k(\beta) .
$$

From (36) we derive $d y=x d \beta+\beta d x+h^{\prime} d \beta$. Substituting this in (37), we obtain

$$
\frac{d x}{d \beta}=\frac{x+h^{\prime}(\beta)}{k(\beta)-\beta} \text {. }
$$

Thus by means of quadratures $x$ is obtained as a function of $\beta$; this together with (36) provides a parametric representation of the cross characteristics. For the principal stress-lines the procedure is the same except that $d y / d x=\tan \theta$ is used instead of (37) while the relation between $s$ and $\beta$ is the same as before. In case of a centered wave $h(\beta)=0$.

It is well known that simple waves are adjacent to regions of constant state; this is 
deduced immediately from the fact that a region of constant $s, \theta$ is mappeed onto a single point in the stress graph.

A simple wave can transform any state $s=s_{1}, \theta=\theta_{1}$ into another state $s=s_{2}$, $\theta=\theta_{2}$ provided either $\theta+F(s)$ or $\theta-F(s)$ (where $F(s)$ is defined in $\left(28^{\prime}\right)$ ) has the same value for the first and second state. By combining a forward and a backward wave and inserting a uniform state in between, any $s_{2}, \theta_{2}$ can be reached; in each wave the envelope of the straight characteristics can still be chosen in various ways.

7. Examples of simple waves. Consider the quadratic yield condition (1) and the forward wave, image of $\theta=F(s)$. Using $\left(28^{\prime}\right)$ and (29):

$$
\theta=F(s) \equiv \arctan \left[s\left(3-s^{2}\right)^{-1 / 2}\right]-\frac{1}{2} \arctan \left[\frac{s}{2}\left(3-s^{2}\right)^{-1 / 2}\right] .
$$

Here the $C^{-}$are straight lines, $\delta=\theta-\varphi$, and as $\sigma_{2}>\sigma_{1}, \tan \varphi$ is given by (30) with + sign. Hence

$$
\delta=\theta-\varphi=\arctan \frac{s}{\left(3-\mathrm{s}^{2}\right)^{1 / 2}}-\frac{1}{2} \arctan \frac{s}{2\left(3-s^{2}\right)^{1 / 2}}-\arctan \frac{\left(12-3 s^{2}\right)^{1 / 2}-s}{2\left(3-s^{2}\right)^{1 / 2}} .
$$

To simplify, we introduce $t=+s\left(3-s^{2}\right)^{-1 / 2}$ and obtain

$$
\delta=\arctan t-\frac{1}{2} \arctan \frac{t}{2}-\arctan \left[\frac{1}{2}\left(4+t^{2}\right)^{1 / 2}-t\right],
$$

which reduces to

$$
\delta=\arctan t-\frac{\pi}{4}, \quad t=\tan \left(\delta+\frac{\pi}{4}\right)
$$

or, reintroducing $s$,

$$
s=\sqrt{3} \sin \left(\delta+\frac{\pi}{4}\right), \quad \theta=F(s) .
$$

Here as $s$ goes from $-\sqrt{3}$, to 0 , to $+\sqrt{3}$, $(t$ goes from $-\infty$, to 0 , to $+\infty), \theta$ goes from $-\pi / 4$, to 0 , to $+\pi / 4$ and $\delta$ from $-3 \pi / 4$ to $+\pi / 4$ (see Fig. 3). Hence in a "complete" wave, $\delta$ varies by $180^{\circ}$. In actual problems a solution is given in such parts of the $x, y$ plane only where the straight characteristics do not intersect; thus parts of complete waves may appear as solutions.

The image of the $\Gamma_{0}^{-}$characteristic $\theta+F(s)=0$ is a backward wave with rectilinear $C^{+}$lines and $\delta=\theta+\varphi=-[F(s)-\varphi]$. Hence this $\Gamma^{-}$wave is the reflected $\Gamma^{+}$wave considered above and $\delta=\pi / 4-\arctan t$, etc. We see that in a foward wave as $\delta$ increases, the mean pressure as well as $\theta$ increase in a monotonous way. In a backward wave the opposite is true.

Next, we compute the cross characteristics for the backward wave $\theta=-F(s)$, $\delta=\pi / 4-\arctan t$. With notations as in (26), (36), and (37), we obtain:

$$
\begin{gathered}
\beta=\tan \delta=\tan \left(\frac{\pi}{4}-\arctan t\right)=\frac{1-t}{1+t}, \quad t=\frac{1-\beta}{1+\beta} . \\
\tan (\theta-\varphi)=\tan \alpha^{\prime}=-\frac{t^{2}+t+2}{t^{2}-t+2}=-\frac{\beta^{2}+\beta+2}{2 \beta^{2}+\beta+1}=k(\beta) .
\end{gathered}
$$


We may then continue as explained above. For the particular case of a centered wave, where $h(\beta)=0, \beta=y / x$, the differential equation of the cross characteristics becomes

$$
\tan \alpha^{\prime}=\frac{d y}{d x}=-\frac{y^{2}+x y+2 x^{2}}{2 y^{2}+x y+x^{2}}
$$

which can be integrated easily. One finds, using polar coordinates, $r, \delta$ (Fig. 5)

$$
r^{2} \cos \left(\frac{\pi}{4}-\delta\right)=\text { const! }
$$

Let us conclude this consideration of the quadratic condition by deriving a simple characterization of the stress tensor which holds if at each point of the wave we use a

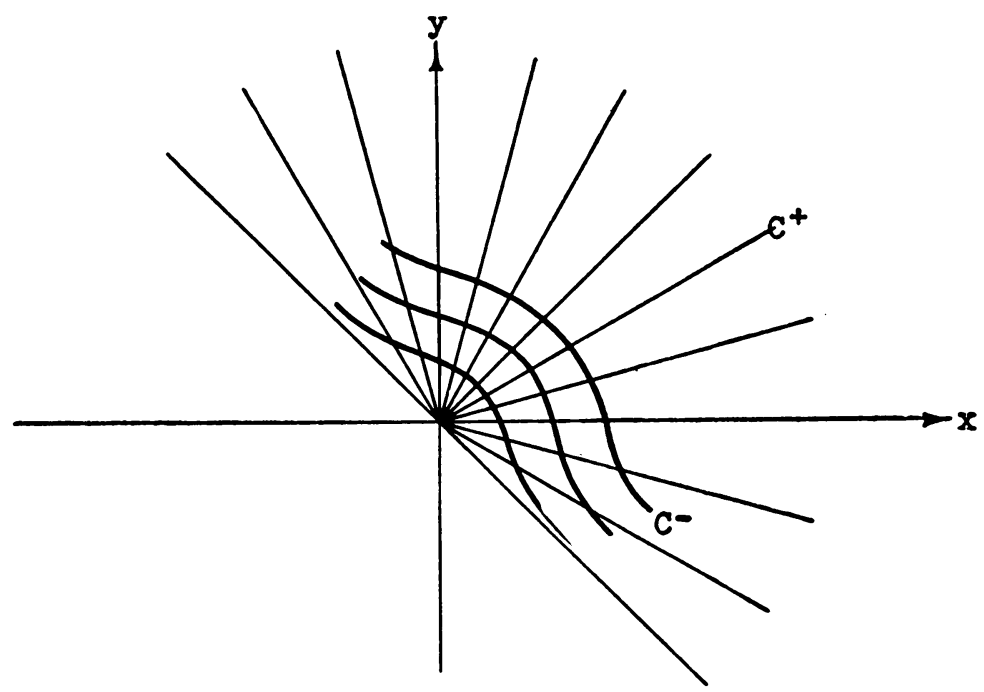

FIG. 5. Quadratic limit. Complete centered wave. Straight characteristics and cross characteristics. .

coordinate system $x^{\prime}, y^{\prime}$ where $x^{\prime}$ is the direction of the straight line through this point, $y^{\prime}$ the perpendicular direction. The stress tensor is then given by $\sigma_{x^{\prime}}, \sigma_{y^{\prime}}, \tau_{x^{\prime} y^{\prime}}$. Denote by $\nu_{1}, \nu_{2}$ the two principal directions at a point and let $\sigma_{x^{\prime}}=\sigma, \sigma_{y^{\prime}}=\bar{\sigma}, \tau_{x^{\prime} y^{\prime}}=\tau$; then $\left(\nu \nu_{1}\right)=\delta-\theta=\varphi$ and we find

$$
\sigma=\sigma_{1} \cos ^{2} \varphi+\sigma_{2} \sin ^{2} \varphi, \quad \tau=\left(\sigma_{2}-\sigma_{1}\right) \sin \varphi \cos \varphi .
$$

Using primes to denote derivatives, we find from (1), $\left(16^{\prime}\right),(34)$ :

$$
\begin{gathered}
\cos ^{2} \varphi=\sigma_{1}^{\prime} / 2 K, \quad \sin ^{2} \varphi=\sigma_{2}^{\prime} / 2 K \\
\sigma=\frac{1}{2 K}\left(\sigma_{1} \sigma_{1}^{\prime}+\sigma_{2} \sigma_{2}^{\prime}\right)=\frac{1}{4 K} \frac{d}{d s}\left(\sigma_{1}^{2}+\sigma_{2}^{2}\right)=\frac{1}{4 K} \frac{d}{d s}\left(\sigma_{1} \sigma_{2}\right) \\
=\frac{1}{4 K} \frac{d}{d s}\left[\frac{4 K^{2}}{3}\left(s^{2}-1\right)\right]=\frac{2 K}{3} s, \quad \tau=\frac{2 K}{3}\left(3-s^{2}\right)^{1 / 2} .
\end{gathered}
$$


Also,

$$
\bar{\sigma}=\frac{1}{2 K}\left(\sigma_{1} \sigma_{2}^{\prime}+\sigma_{2} \sigma_{1}^{\prime}\right)=\frac{1}{2 K} \frac{d}{d s}\left(\sigma_{1} \sigma_{2}\right)=2 \sigma .
$$

Hence under quadratic yield condition the following simple relations hold for the stress tensor all over in a simple wave region

$$
\sigma^{2}+\tau^{2}=\frac{4}{3} K^{2}, \quad \bar{\sigma}=2 \sigma .
$$

Parabola limit. Using (31) and $s / a=t$, we consider the image of the $\Gamma^{-}$characteristic

$$
\theta=-\arcsin t=\arccos t-\frac{\pi}{2}
$$
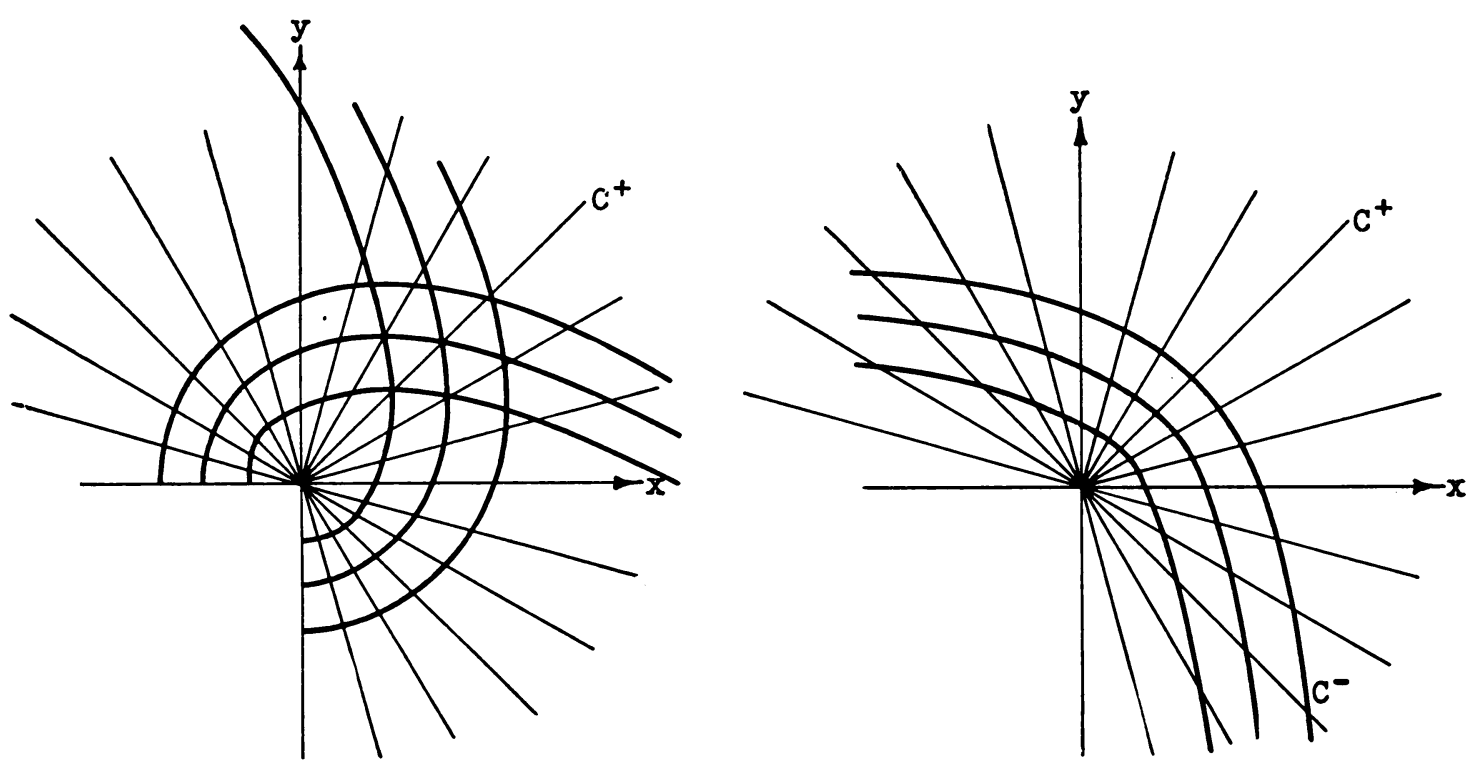

Fig. 6a. Parabola limit. Straight characteristics and cross characteristics.

FIG. 6b. Parabola limit. Lines of principal stress.

From

$$
\tan \varphi=\left(\frac{a-s}{\mathrm{a}+s}\right)^{1 / 2}=\left(\frac{1-t}{1+t}\right)^{1 / 2}=\tan \left(\frac{\pi}{4}+\frac{\theta}{2}\right),
$$

we conclude that

$$
\varphi=\frac{1}{2}\left(\theta+\frac{\pi}{2}\right), \quad \delta=\varphi+\theta=\frac{3 \theta}{2}+\frac{\pi}{4}
$$

and thus:

$$
\theta=\frac{2}{3}\left(\delta-\frac{\pi}{4}\right), \quad s=a \cos \left(\theta+\frac{\pi}{2}\right)=a \cos \frac{\pi+2 \delta}{3}
$$


Here the maximum interval for $\theta$ equals $180^{\circ}$ and for $\delta$ it equals $270^{\circ}$; as $\theta$ goes from $\pi / 2$ through 0 to $-\pi / 2, \delta$ goes from $\pi$ through 0 to $-\pi / 2$.

We compute now the cross characteristics for a centered wave. From (50), using polar coordinates $r$ and $\delta$ we obtain (Fig. 6a)

$$
\begin{gathered}
\frac{r d \delta}{d r}=-\tan 2 \frac{-}{\varphi}=-\tan \left(\frac{\pi}{2}+\theta\right)=-\tan \frac{2 \delta+\pi}{3} \\
r=r_{0}\left(\sin \frac{2 \delta+\pi}{3}\right)^{-3 / 2} .
\end{gathered}
$$

We finally compute for the same example the principal stress lines. For the $\xi$-lines, the lines that make the angle $\theta$ with the $x$-direction, we have, in polar coordinates (Fig. 6b)

$$
\frac{r d \delta}{d r}=-\tan (\delta-\theta)=-\tan \left(\frac{\delta}{3}+\frac{\pi}{6}\right)
$$

or

$$
r=r_{0}\left[\sin \frac{2 \delta+\pi}{6}\right]^{-3}
$$

and for the $\eta$-lines

$$
r=r_{0}\left[\cos \frac{2 \delta+\pi}{6}\right]^{-3}
$$

These examples may suffice.

Simple waves are widely used in the solution of actual problems.

\section{REFERENCES}

1. H. Geiringer, (a) Linear differential equations of the plane stress problem of a perfect plastic body, (b) Parabola-yield condition for the perfect plastic body, (c) Simple wave solutions for the plane stress problem of the perfect plastic body, Bull. Amer. Math. Soc., 56, 38-39 (1950).

2. P. G. Hodge, Jr., An introduction to the mathematical theory of perfectly plastic solids, Graduate Division of Applied Mathematics, Brown University, Providence, R. I., (1950).

3. W. Jenne, Räumliche Spannungsverteilungen in festen Körpern bei plastischer Deformation, Z. angew, Math. Mech. 8, 1-27 (1928).

4. R. v. Mises, (a) Three remarks on the theory of the ideal plastic body, Reissner Anniversary Volume, J. W. Edwards 1949, 415-429. (b) Mechanik der plastischen Formänderung von Kristallen, Z. angew. Math. Mech. 8, 161-185 (1928).

5. W. Prager, Recent developments in the mathematical theory of plasticity, J. Appl. Phys. 20, 235-241 (1949).

6. W. W. Sokolovsky, (a) Plastic plane stressed state according to Mises, Doklady, 51, 175-178 (1946).

(b) Plastic plane stressed state according to Saint Venant, Doklady, 51, 421-424 (1946). (c) The theory of plasticity, an outline of work done in Russia, J. Appl. Mech. 13, A1-A10 (1946). 